\title{
Aproximaciones al rol de los planificadores regionales de la Iniciativa para la Integración de la Infraestructura Regional Suramericana (IIRSA)
}

\author{
Damián Lobos. IPICB, Córdoba, Argentina \\ Klaus Frey. Universidade Federal do АBC (UfABC), Santo André, Brasil.
}

RESUMEN | En el presente artículo se analiza el rol central que vienen cumpliendo los planificadores regionales alineados con la visión del regionalismo abierto cepalino en la actual estrategia de inserción de América Latina en el capitalismo global, en vista de su sujeción a los imperativos mundiales del capital transnacionalizado. Para ello se enfoca la atención en los instrumentos metodológicos de la Planificación Territorial Indicativa (PTI) de la Iniciativa para la Integración de la Infraestructura Regional Suramericana (IIRSA), entendiendo a esta última como institución reproductora del regionalismo abierto de la CEPAL desde su propuesta general de integración física regional. Se exponen cuatro secciones donde se desarrollan la emergencia del regionalismo abierto cepalino como estrategia espacial del Estado y la relevancia de IIRSA en torno a la reproducción del mismo, para luego analizar el rol que desempeńan en la misma los planificadores regionales desde la creación de consenso por medio de la PTI, hasta la creación de territorialidades particulares.

PALABRAS CLAVE | planificación territorial, integración regional, geografía económica, reescalamiento.

ABSTRACT I In this article the central role played by territorial planners is analyzed together with the vision of the Economic Commission for Latin America and the Caribbean (ECLAC) concerning Open Regionalism in the current strategy related to the insertion of Latin America in the global capitalism, based on its subjection to the imperatives of trans nationalized capital. It focuses on the methodological tool of Indicative Territorial Planning (Planificación Territorial Indicativa, PTI) as part of the Initiative for the Integration of the Regional Infrastructure of South America (Iniciativa para la Integracion de la Infraestructura Regional Suramericana, IIRSA), the latter being understood as an institution reproducing ECLAC's open regionalism, in the light of its general proposal on regional physical integration. Four sections are presented where the emergence of ECLAC's concept of open regionalism as a state space strategy and the relevance of IIRSA in its promotion and dissemination are developed, and then to analyze the role played by territorial planners from seeking consensus by PTI to the creation of particular territorialities.

KEYWORDs | territorial planning, regional integration, economic geography, rescaling. 
Este trabajo parte de la idea preliminar de que los planificadores regionales enfilados tras la visión del regionalismo abierto cepalino vienen desempeńando un rol clave en la actual estrategia de inserción de América Latina en el capitalismo global, la cual se encuentra sujeta a los imperativos mundiales del capital transnacionalizado. Se plantea que este rol consiste en viabilizar los intereses de los agentes del capital global (empresas transnacionales y fondos financieros) por sobre los capitales y gobiernos nacionales y, sobre todo, por sobre los sectores populares. En este escenario, los Estados nacionales terminan asumiendo un papel puramente pasivo en la toma de decisiones de lo que implica planificación de escala regional transnacional, pero profundamente activo en la implementación de la misma. La gravedad de esta carencia se hace patente en el carácter represivo que han venido adoptando los Estados en los conflictos locales generados por la implementación de los proyectos de la Iniciativa para la Integración de la Infraestructura Regional Suramericana (IIRSA), como son los caso del conflicto Cachuel-Esperanza en Brasil; las sistemáticas persecuciones, asesinatos y expulsiones de campesinos en la región oriental del Paraguay; las muertes y persecuciones de mineros en el Cajamarca peruano; las puebladas de resistencia contra las mineras en la cordillera argentina, entre muchos etcéteras.

Bajo estos imperativos, los planificadores regionales hacen uso de procedimientos tecnocráticos particulares que tienen por finalidad la selección, retención y consolidación de determinados discursos, prácticas e identidades. Con miras a discutir algunos de estos aspectos se propone entender a los planificadores regionales desde el concepto de tecnocracia, en el sentido de actualización del imperativo de la racionalidad técnica de los economistas neoliberales por sobre otras consideraciones económico-espaciales.

No es intención de este trabajo abordar de lleno estas cuestiones, sino proponer líneas de discusión que rocen algunos aspectos que se consideran relevantes. Para ello es que se hace foco en los instrumentos metodológicos de la Planificación Territorial Indicativa (PTI) de la Iniciativa para la Integración de la Infraestructura Regional Suramericana (IIRSA), entendiendo a esta última como institución reproductora del regionalismo abierto de la Comisión Económica para América Latina y el Caribe (CEPAL) desde su propuesta general de integración física regional.

Con este panorama es que se presentan las cuatro siguientes secciones. Se comienza (apartado 1) con una exposición del contexto de emergencia de IIRSA y del regionalismo abierto cepalino desde la perspectiva de las estrategias espaciales del Estado latinoamericano, en un contexto de revalorización del capital; luego (apartado 2) se focaliza en el papel de la tecnocracia de planificadores regionales de IIRSA y, particularmente, en la producción de los instrumentos de Planificación Territorial Indicativa (PTI), para después (apartado 3) trazar una delimitación de la territorialidad propia de IIRSA. Se concluye con un resumen y algunas propuestas para guiar el debate sobre el rol de los planificadores regionales de IIRSA en la selección y sostenimiento del modelo extractivo-exportador en Suramérica en la actualidad. 


\section{Regionalismo abierto como estrategia estatal}

En esta sección se parte de la noción de selectividad espacial de la circulación del capital, centrándose en el rol estratégico de los Estados para influenciar la misma. Luego se plantean dos momentos de reconfiguración de las estrategias espaciales de los Estados latinoamericanos: el enfoque liberalizador del Consenso de Washington, y el regionalismo abierto de la CEPAL. Por último, se plantea la emergencia y relevancia de IIRSA como agente reproductor de la estrategia del regionalismo abierto cepalino.

Desde un abordaje estratégico-relacional (Jessop, 2008), la forma diferencial en la que toma cuerpo el capital en cada territorio produce selectividades también diferenciales hacia la aplicación de estrategias espaciales por parte de los actores socioeconómicos implicados. Por un lado, los ajustes espaciales del capital global ${ }^{1}$ implican una compleja vinculación entre espacialidades capitalistas diferentes dentro del micronivel de las economías particulares. Por otro lado, en estos procesos de reconfiguración espacial del capital global desempeñan un rol clave las estrategias de acumulación que los diferentes Estados locales aplican. Brenner (2003) define "estrategia espacial del Estado" como la puesta en acto por parte de este de su capacidad de influencia sobre la geografía de la acumulación y de la regulación, modelando "las geografías del desarrollo industrial, de la inversión en infraestructura y de las conflictividades políticas, en procura de ajustes espaciales o coherencia estructural" (p. 204).

En este sentido, los procesos de dislocación espacial (y/o temporal) del capital global impactan en el espacio no solo dependiendo de la posición de los diferentes territorios en el esquema global de circulación del mismo. Su impacto diferencial también depende de la aplicación de estrategias estatales concretas de modificación, modelación y trasformación de los atributos espaciales del capital, que tienen como finalidad la absorción y/o expulsión del mismo.

En los ańos posteriores a la Segunda Guerra Mundial, el desarrollo de las tecnologías de la producción y la explotación excesiva de la fuerza de trabajo en las economías capitalistas centrales provocaron una estrepitosa caída de la tasa de ganancia en la producción de mercancías industriales de consumo masivo (Benko, 2002). La respuesta a la crisis de acumulación fordista en los países capitalistas centrales implicó una flexibilización y deslocalización del capital acumulado hacia fuera de sus economías. Esto redundó (como contrapartida) en la masiva inyección de capitales, en forma de inversión extranjera directa (IED) o de fondos de inversión.

En el caso latinoamericano, el arribo de esta masa de capital financiero internacional se observa directamente vinculado, en las décadas de los ochenta y noventa, a la aplicación de una serie de reformas de liberalización y apertura económica, conocidas como el Consenso de Washington. El mismo tuvo como principal estrategia de reproducción la generalización de prácticas políticas tecnocráticas de tinte neoliberal, principalmente sostenidas por el Banco Mundial y el Fondo Monetario Internacional (Silva, 1997). Las premisas centrales del mismo fueron la liberalización de

Harvey (2005) caracteriza el ajuste espacio-temporal como aquel proceso de dislocación de los excedentes de trabajo y capital de una economía que busca influir sobre sus efectos críticos. 
la circulación del capital entre los espacios nacionales y la puesta en primacía del inversor capitalista por sobre el intervencionismo estatal. Como estrategia estatal, el enfoque liberalizador asume la noción de ventajas comparativas naturales; esto es, ante la eliminación de toda traba y distorsión por parte del Estado, el inversor privado seleccionaría la localización de su capital según atributos naturales de los territorios. La escala de acumulación no tiene fronteras y la economía política de los Estados asume por única finalidad la generación de un ambiente atractivo para la inversión privada (De Mattos, 2004).

Hacia fines de la década de los noventa se empieza a observar una nueva matriz en la circulación internacional del capital, donde adquieren una importante (re) valorización (financiera) los productos primarios y/o materias primas ${ }^{2}$. Los nuevos flujos norte-sur adoptan la forma de nuevos mercados internacionales, en el sentido de diversificación de la inversión directa y nuevas selectividades en la financiación. En América Latina, estos nuevos mercados han tomado cuerpo en las actividades extractivas (metales, biocombustibles, oleaginosas) y en la producción de materia prima para la exportación. De este modo, el subcontinente se convierte en un partícipe clave del nuevo régimen de circulación global del capital. Svampa (2008) plantea para gran parte de los países de América Latina una situación "caracterizada por la generalización de un modelo extractivo-exportador (...) basado en la extracción de recursos naturales no renovables, la extensión del monocultivo, la contaminación y la pérdida de biodiversidad" (p. 4).

En paralelo, un nuevo reflujo de movilizaciones populares, sumado a los limitados beneficios que el enfoque liberalizador les trajo a los capitalistas nacionales latinoamericanos ${ }^{3}$, supuso un inicial cuestionamiento y un paulatino abandono de la estrategia del Consenso de Washington por parte de los Estados latinoamericanos. Con ello, el Banco Mundial (вм) y el Fondo Monetario Internacional (FмI) fueron perdiendo centralidad relativa en los ámbitos decisorios estatales ${ }^{4}$, donde comenzó a emerger nuevamente el nombre de la CEPAL.

Desarrollado a comienzos de los noventa, el regionalismo abierto cepalino implica una estrategia espacial estatal caracterizada por la continuidad de los imperativos del consenso de Washington, pero en tanto readaptación estratégica del mismo, al proponer a la región latinoamericana como una escala en sí misma. Gudynas (2005) destaca que el regionalismo abierto difiere de la simple apertura del comercio y la promoción no discriminada de las exportaciones, en cuanto asume la relevancia de

2 Proveniente de las interrelaciones entre la apertura de China hacia el consumo internacional, las políticas de preservación de reservas de petróleo y metalíferas en las economías capitalistas centrales (sumadas a las incursiones militares para asegurar el control de reservas ajenas) y el avance en los desarrollos de las agrotecnologías (principalmente en energía y combustibles).

3 La resultante, durante los ańos ochenta y noventa, de la aplicación en América Latina del enfoque liberalizador fue la internacionalización de su capacidad productiva instalada, una limitada inversión directa en industria y la toma compulsiva de deuda por parte de los agentes privados nacionales y del Estado, las mismas que generaron crisis de solvencia en las economías y consecuentes presiones inflacionarias.

4 Frey (2008) destaca cómo las reconceptualizaciones históricas de "desarrollo" y su uso selectivo -a partir de los años noventa- de los conceptos de local development y good governance son utilizados por el Banco Mundial como estrategias discursivas de relegitimación internacional. 
los acuerdos de integración previamente concertados, la cercanía geográfica de los Estados participantes y la afinidad cultural de los mismos. Las ventajas comparativas son regionales, lo que implica una estrategia espacial de los Estados nacionales para captar inversiones por medio de la extensión relativa de sus mercados de consumo y la conectividad de su diversidad económico-productiva.

Con el desarrollo de los procesos de integración regional, en los años ochenta toma un importante impulso la planificación en infraestructura subregional desarrollada principalmente en el cono sur (Mercosur), donde se destacan los corredores bioceánicos (ruteros y ferroviarios) y la hidrovía Paraguay-Paraná. En los años noventa hubo muchos tratados de integración relevantes, casi siempre impulsados por la CEPAL o algún otro organismo subregional ${ }^{5}$, pero los mismos no lograron hacer del regionalismo abierto una estrategia generalizada y con fuerza en todos los Estados. Las restricciones al financiamiento internacional de los Estados latinoamericanos -tras las crisis de las deudas nacionales- fueron el principal factor que los hiciera alejarse de los preceptos de la perspectiva liberalizadora.

Los principales antecedentes en lo que hace a planificación territorial en América Latina en el siglo xx fueron, por su envergadura y complejidad, las políticas brasileñas sobre infraestructuras de transporte, donde destaca el pionero programa Brasil em $A c ̧ a \tilde{a} o^{6}$. Cocco, Monié y Silva (2000) refieren en torno a este último;

En un país de dimensiones continentales como Brasil, con fronteras de expansión agrícola todavía abiertas y espacios geográficos aún inexplorados, las estrategias destinadas a consolidar y ampliar las posibilidades de vinculación entre las diferentes regiones, y de estas con los mercados externos, representan aspectos claves de las nuevas modalidades económicas ensayadas por el gobierno para su inserción competitiva en la economía mundial. En tal sentido, el programa Brasil em Ação manifiesta la preocupación de las autoridades federales frente a los nuevos desafíos abiertos por la globalización, indicando medidas concretas a ser implementadas en el transcurso de los próximos años en diferentes ámbitos del País. Revela además la concepción que guía y articula los diversos proyectos, enfatizando la necesidad de considerar las potencialidades existentes en cada región, tanto en términos de producción cuento de circulación y transporte. (p. 21)

Pero no fue hasta el desarrollo de los bloques regionales hacia comienzos del siglo xxi que el regionalismo abierto cepalino toma real relevancia como estrategia espacial de los Estados nacionales latinoamericanos. En la cumbre de presidentes de Suramérica de Brasilia de 2000 se asume la planificación regional como uno de los temas prioritarios para una agenda conjunta de integración física entre los países. En el Comunicado de Brasilia se explicita que "integración y desarrollo de la infraestructura física son dos líneas de acción que se complementan” (punto 37). Para la consecución de estas finalidades, se propone la profundización del trabajo

5 Tal vez el más relevante sea el tratado de Asunción de 1991, que crea el Mercosur, mayor ejemplo subregional y arquetipo del proyecto de regionalismo abierto.

6 Posteriormente articuladas en el Programa de Aceleração do Crescimento (PAC), al que en años recientes le suman el Plan Plurianual (PPA) 2008-2011 y el Plan Nacional de Logística de Transportes (PNLT). 
en el ámbito de obras de interés bilateral y subregional, al tiempo que se considera que "la financiación de los proyectos de infraestructura de integración deberá ser compartida por los gobiernos, por el sector privado y por las instituciones financieras multilaterales" (punto 38). Dentro de este marco emergen los llamados megaproyectos de planificación de conectividad física regional, como son los casos de la Iniciativa para la Integración de la Infraestructura Regional Suramericana (IIRSA) y el Plan Puebla-Panamá en el año 2004.

En la actualidad, la planificación y el desarrollo de infraestructura de escala regional en Latinoamérica están entre los principales ejes discursivos de los organismos regionales y mundiales ${ }^{8}$.

\section{Tecnocracia de planificadores regionales de IIRSA y metodologías de planificación como constructoras de consenso}

En esta sección se identifica la posición que ocupan y la práctica que ejercen los tecnócratas planificadores regionales de IIRSA, como agentes de materialización del regionalismo abierto cepalino, entendido este como estrategia espacial del Estado en el actual contexto de valorización financiera de los bienes primarios en la subregión. Se parte de una lectura de las relaciones conflictivas internas al proyecto IIRSA, y de las necesidades de articulación que emanan de las mismas. Luego se expone la tecnocracia de planificadores regionales de IIRSA en cuando continuación y ruptura con la tecnocracia de economistas neoliberales de los ańos noventa. Por último, se presentan los principales instrumentos metodológicos de la Planificación Territorial Indicativa.

Siguiendo a Silva (2010), la tecnocrática es una ideología política que consiste en la ponderación de la preservación del orden social y de la administración científica de los asuntos de Estado, por sobre los derechos individuales. La misma asume un conjunto de principios como verdaderos y universales, por cuanto el buen gobierno o gobierno correcto requiere de la aplicación de políticas coherentes a ellos; la aplicación y producción de normatividad debieran provenir de una derivación de esos preceptos científicos hacia ámbitos de aplicabilidad gubernamental. La consecuencia es una presunta cientifización de la política y de la acción pública. En otro trabajo, Silva (1997) destaca el vínculo entre el aislamiento burocrático de los economistas

El Plan Puebla Panamá (ppr) es un foro de cooperación intergubernamental integrado por nueve países mesoamericanos, creado en el año 2004. Tiene por finalidad la gestión y ejecución de proyectos orientados a la extracción de recursos naturales de Mesoamérica, así como la implantación de vías para interconectar los dos océanos y facilitar la exportación de la producción y su comercialización internacional con los recursos obtenidos en estos países. Es promovido y principalmente financiado por el Banco Interamericano de Desarrollo (BID), el Banco Centroamericano de Integración Económica (BCIE) y la Corporación Andina de Fomento (CAF). Con el ingreso de Colombia en 2009 se cambió su nombre al de Proyecto Mesoamérica.

8 Destacan la serie de trabajos de la CEPAL, que confluyen en el informe "Infraestructura para la integración regional" del año 2011; el voluminoso manual desarrollado por CAF "La infraestructura para el desarrollo integral del América Latina”, de 2011; y el multicitado estudio de Fay y Morrison para el Banco Mundial, "Infraestructura en América Latina y el Caribe. Acontecimientos recientes y desafíos principales", de 2007. 
neoliberales y la aplicación de los preceptos del enfoque liberalizador en las nuevas democracias latinoamericanas, diciendo que para

facilitar la aplicación de los programas de ajuste estructural, numerosos gobiernos democráticos han tratado conscientemente de aislar a los equipos económicos de las presiones directas provenientes de la sociedad. Como consecuencia de esto, en varios países los tecnócratas han obtenido en la actualidad un alto grado de "autonomía relativa” frente a empresarios, sindicatos y partidos políticos, ampliando con esto aún más su espacio de maniobra en la toma de decisiones. (p. 71)

Los desarrollos del paradigma empresarial ${ }^{9}$ norteamericano de los años noventa, particularmente en la línea de Michael Porter (principal intelectual orgánico del empresarialismo monopolista norteamericano $)^{10}$, consisten en una visión global y multinacional de la economía, articulada y organizada en cadenas de valor comandadas por grupos estratégicos. Entre los trabajos de Porter destacan los referidos a las ventajas y estrategias empresariales competitivas y el concepto de "cluster de negocios"11, posteriormente generalizado en el pensamiento empresarial anglosajón y europeo.

Mientras este paradigma tecnocrático neoliberal se concentraba en el accionar de las empresas, los desarrollos de la Nueva Geografía Económica (NGE) han conseguido construir, a partir de las mismas premisas, un abordaje territorial para la intervención estatal a partir de una similar, pero más elaborada noción de competitividad. Como plantea su autor de cabecera Paul Krugman ${ }^{12}$, la NGE se encuentra particularmente interesada en los aspectos territoriales vinculados a la creación de los clusters empresariales: "the defining issue of the new economic geography is how to explain the formation of a large variety of economic agglomeration (or concentration) in geographical space" (Fujita \& Krugman, 2004, p. 140).

La hipótesis central de la NGE es que existen tendencias hacia la (re)localización del capital en el espacio (clustering) y que esas tendencias responden a los rendimientos crecientes de los distintos territorios. Estos rendimientos crecientes territoriales pueden provenir de condiciones naturales (acceso a recursos naturales, distancias físicas entre locaciones, factores climáticos) o artificiales

9 Por "paradigma tecnológico empresarial", Alain Lipietz (1994) comprende el "modelo o paradigma de cómo se produce, cómo se organiza la producción” (p. 2).

10 Profesor de la escuela de negocios de Harvard, defensor de la libre empresa e influyente crítico de toda forma de bienestarismo, Porter ha sido uno de los principales opositores a las reformas al sistema de salud norteamericano propuestas por la bancada republicana y rechazada por la mayoría demócrata en 2013. Su campaña en contra de la propuesta republicana consistió en una fuerte presencia en medios de comunicación, contacto con políticos conservadores y corporaciones empresariales farmacéuticas y de seguridad social, y en la producción de artículos y libros sobre la temática, donde se destaca su publicación en coautoría con JimYong Kim y Paul Farmer "Redefining global health-care delivery" (The Lancet, 2013). Para una crítica a los planes focalizados en zonas periurbanas, véase Porter (2000).

11 Se denomina "cluster de negocios" a una concentración de empresas e instituciones interconectadas propias de un área particular de producción. Esta concentración de empresas tiene como principal requisito la adquisición de ventajas competitivas propias del sector particular (como cercanía y acceso a trabajo barato y a recursos naturales, articulación con esquemas logísticos de circulación, regímenes normativos que viabilicen cierto tipo de producción, etcétera).

12 Premio Nobel de Economía 2008, profesor de Princeton y columnista del New York Times. 
(path-dependency). El proceso resultante es la generación de economías de aglomeración (regiones, centros urbanos, redes de comercio internacional) que concentran capital y lo distribuyen diferencialmente. Los tecnócratas que adhieren a estos preceptos siempre tuvieron presente que el pensamiento que estaban desarrollando sería funcional en cuanto estrategia espacial tecnocrática del Estado en el contexto de la internacionalización monopolista del capital poscrisis de los años setenta. Krugman ${ }^{13}$ escribió al respecto en 2004:

The sort of economy envisaged by the models in the new economic geography should be a prime target for government intervention. There is no presumption there that the market will get it right. Moreover, the models suggest that under some circumstances, small policy interventions can have large and perhaps lasting effects. Finally, because cumulative processes of concentration tend to produce winners and losers, perhaps at the level of nations, there is an obvious incentive for policy makers to try to ensure that their nation emerges as one of the winners. (Fujita \& Krugman, 2004, p. 156)

La cristalización de este objetivo político vino con el cambio de siglo de la mano de los trascendentales informes "Perspectiva territorial de la OCDE" (2001) e "Informe sobre el Desarrollo Mundial 2009: Una nueva geografía económica”, del Banco Mundial (2008).

Como consecuencia de la pérdida de legitimidad que el enfoque liberalizador tuvo tras la crisis de la deuda de los países de la región, se desarrolló una fuerte crítica a la tecnocracia de economistas neoliberales y a su aislamiento burocrático. A partir de allí, la nueva modalidad que ha adoptado la intervención gubernamental se basa en el principio del consenso, habiendo dejado de lado tanto la idea del aislamiento burocrático como la de la cientifización de la política. La política del consenso impone nuevos imperativos a la administración pública. En el caso particular de IIRSA, la planificación de sus proyectos de infraestructura debiera emanar de una lectura consensuada de las finalidades de los distintos territorios y comunidades locales receptoras, lo que implicaría asumir un empoderamiento de las racionalidades locales como productoras de legitimidad.

Sobre los antecedentes de los modelos de planificación propios de IIRSA, Raúl Zibechi, referenciando a Porto Gonçalves, plantea:

el origen teórico político de IIRSA y del Plan Puebla Panamá se encuentran en dos estudios. El primero fue "Infraestructure for Sustainable Development and Integration of South America”, realizado por Eliézer Batista da Silva en 1996 para la Corporación Andina de Fomento, la Vale do Rio Doce, el Business Council for Sustainable Development Latin America, el Bank of America y la Companhia Auxiliar de Empresas de Mineração. El segundo se denominó "Estudo sobre Eixos Nacionais de Integraçáo e Desenvolvimento", en 1997, promovido por el BNDES, el Ministerio de Planeación, el Banco $A B N$ Amro, la multinacional estadounidense

13 El artículo en referencia presenta el resumen de una conversación sostenida por los autores en San Juan, Puerto Rico, en noviembre de 2002, con el apoyo de un interlocutor. De allí que el texto citado corresponda a solo uno de ellos. 
Bechtel, Consorcio Brasiliana y Booz Allen \& Hamilton do Brasil Consultores. La lectura de quienes financian estos trabajos permite deducir los intereses que encarnan. El concepto de ejes de integración y desarrollo sustituye al de región como núcleo de la acción gubernamental, privilegiando los flujos por sobre los territorios habitados por pueblos y naciones. (pp. 201-202)

Con el andar de la iniciativa IIRSA, los equipos de planificación han desarrollado y sistematizado un cierto know-how sobre la forma correcta y/o preferible de llevar a cabo los procesos de planificación y toma de decisiones sobre las obras de infraestructura. La Planificación Territorial Indicativa (PTI), denominación que desde IIRSA se le otorga al conjunto de metodologías de elaboración, caracterización, comparación, selección y aplicación de sus proyectos (IIRSA 10 AÑOS DESPUÉS: sus logros y desafíos, 2010), busca ser una suerte de explicitación y sistematización de las prácticas de planificación llevadas adelante por el Comité de Coordinación Técnica (сСт) de IIRSA ${ }^{14}$. Durante la primera década de su existencia, el contenido de la PTI cambió mucho, pero ya en el año 2009 se presentaron versiones finales de algunos de sus compendios metodológicos. Vamos a destacar los tres que consideramos más relevantes ${ }^{15}$ :

- Visiones de negocios: no se encuentran definidas con precisión en ningún documento, pero serían algo así como un estudio de potencialidades económicas para cada Eje de Integración y Desarrollo (EID). Las distintas visiones de negocios fueron financiadas y presentadas por las instituciones del ССт, las que encargaron los estudios a consultores de gran reconocimiento en el ámbito privado. Las mismas fueron primeramente presentadas en los años 2002-2004 y luego revisadas y reformuladas en 2007-2008. Estos informes se basan principalmente en información secundaria y suelen ser versiones extendidas de estudios privados sobre costos de inversión y servicios logísticos realizados previamente.

- Análisis del Potencial de Integración Productiva y Desarrollo de Servicios Logísticos de Valor Agregado (IprLg): herramienta metodológica para evaluar el potencial de integración productiva y de desarrollo de servicios logísticos de valor agregado en el área de influencia de grupos de proyectos. La versión definitiva del año 2009 fue preparada por el Instituto para la Integración de América Latina y el Caribe (BID/INTAL) y desarrollado por dos consultores privados. Esta metodología fue aplicada en el grupo 3 del Eje de Capricornio (2009), en el grupo 5 del Eje Interoceánico Central (2009) y en el grupo 5 del Eje Andino (2010).

- Metodología de Evaluación Ambiental y Social con Enfoque Estratégico (EASE): herramienta de evaluación ambiental que permite identificar, caracterizar y evaluar las consecuencias ambientales y sociales, los riesgos y oportunidades de desarrollo sostenible asociadas al territorio, orientando así el proceso de toma

$14 \overline{\text { El mismo se define como la instancia de apoyo técnico y financiero del proyecto iIRSA, y se }}$ encuentra integrado por el Banco Interamericano de Desarrollo (BID), la CAF, el Banco de Desarrollo de América Latina y el Fondo Financiero para el Desarrollo de la Cuenca del Plata (Fonplata).

15 Listado más amplio en sección "Documentos oficiales IIRSA revisados", inmediatamente antes de Referencias bibliográficas. 
de decisiones en el marco de la planificación de los grupos de proyectos. La versión definitiva de 2009 estuvo a cargo de la Dirección de Medio Ambiente, Corporación Andina de Fomento (DMA-CAF, Caracas) ${ }^{16}$.

\section{La territorialidad del IIRSA}

En esta sección se plantean algunas líneas de caracterización de la territorialidad propia del IIRSA. Luego de proponer la necesidad del desarrollo de un concepto de territorialidad propio de IIRSA, se exponen las tres delimitaciones territoriales identificadas a partir de los instrumentos de la PTI (el territorio latinoamericano, los Ejes de Integración y Desarrollo y las áreas de influencia de los Grupos de Trabajo) $\mathrm{y}$ algunos apuntes generales en torno a las mismas.

Desde la perspectiva de los capitales internacionales, IIRSA implica una relectura estratégica de los Estados nacionales hacia una escalaridad regional sudamericana y un plan de inversiones en obras de conectividad que sirve a los fines de ajuste espacio-temporal. Desde la perspectiva de los Estados de Suramérica, la creación de IIRSA es la posibilidad de abrir nuevos canales de financiamiento para sus economías ${ }^{17}$, cerrados por los acreedores internacionales y las instituciones del enfoque liberalizador. En este sentido es que adquiere fuerza la iniciativa y logra concentrar en su seno una doble agenda: la de los principales proyectos de los Estados nacionales y las de los proyectos de conectividad regional, ambas articuladas bajo los imperativos del capital internacional. Estas agendas están primariamente delimitadas por los requerimientos concretos de la valorización financiera de los territorios que el modelo extractivo-exportador ha venido imponiendo en las últimas dos décadas. Ambas dan cuenta de una nueva lectura del territorio ${ }^{18}$; en sí IIRSA implica una lectura concreta de los territorios, de sus potencialidades diferenciales y de sus dinámicas concretas.

La lectura más acabada del territorio latinoamericano de IIRSA se encuentra en un informe del ССт $^{19}$ aquí extractado, según el cual la territorialidad latinoamericana de IIRSA no responde a la morfología natural del territorio, sino que la misma es

16 Hasta el momento, esta metodología solo ha sido aplicada en la evaluación del circuito turístico binacional de los Grandes Lagos entre Argentina y Chile, perteneciente al grupo 2 del eID del Sur, publicado en el año 2010.

17 En el informe Financiación de proyectos transnacionales de infraestructura en América del Sur (iniciativa IIRSA) se propone la utilización de la herramienta financiera unbundled como estrategia de IIRSA para acceder al financiamiento internacional para proyectos bi- o trinacionales. Por otra parte, en el informe Financiamiento privado de infraestructuras. Estudio de alternativas y experiencias de proyectos de Participación Público-Privada para América del Sur, realizado por la CAF, se proponen como modelos diversas experiencias europeas de participación público-privada (PPP) para proyectos de carácter nacional.

18 Mancano Fernández (2008) plantea que la enorme diversidad de comprensiones y prácticas contenidas en un mismo territorio exterioriza posiciones de clase e intereses materiales concretos, activados en un espacio de disputa material e inmaterial. Toda delimitación territorial (sea de la escala y dimensión que fuere) lleva en su seno la intencionalidad que los distintos agentes productores del espacio buscan imprimirle a un territorio concreto. 
vista como una serie de barreras $^{20}$ que generan aislamientos productivos. En aras de una visión regional de la integración física favorable a una mejor inserción en los mercados extrarregionales, esta morfología debe ser modificada (propuesta estratégica de reducción de costos y aumento de conectividad). La finalidad última sería llegar a la movilidad de personas, bienes, capital y conocimiento en un espacio de fronteras cada vez más permeables.

Ya desde la Planificación Territorial Indicativa, los planificadores regionales de IIRSA desarrollaron (tras un proceso largo y burocrático iniciado en la V Reunión del Comité de Dirección Ejecutiva en 2003) una subdivisión de territorios diferenciados hacia dentro de ese territorio latinoamericano, a los cuales dan el nombre de Ejes de Integración y Desarrollo (en adelante EID). Esta delimitación territorial es definida como una franja multinacional de territorio que incluye una cierta dotación de recursos naturales, asentamientos humanos, áreas productivas y de servicios logísticos (IIRSA 10 AÑOS DESPUÉS, 2011).

En el Plan de Acciones de Montevideo de 2000 se explicitan seis criterios que debieran ser considerados a la hora de delimitar los EID, a saber: a) cobertura geográfica de países y regiones, b) flujos existentes y c) potenciales, d) volumen de inversiones recientes, en ejecución y proyectadas en el corto plazo, e) interés y grado de participación del sector privado y, finalmente, f) el grado de sustentabilidad ambiental y social de los proyectos. Estos diversos criterios provocaron que la delimitación de los EID haya variado mucho con el correr de los años y serán las versiones definitivas de las visiones de negocios el principal instrumento de referencia para su identificación. Es en la segunda mitad de la década cuando se encuentra consolidada la interpretación de la existencia de nueve eID que abarcan diferencialmente la mayor parte de la geografía del subcontinente. La delimitación respondió a patrones diversos particulares de cada Visión de Negocios, poniendo el foco, en algunos casos, en cierto recurso compartido (de la hidrovía Paraguay-Paraná); en otros, en la existencia de alguna barrera natural (del Amazonas, interoceánico central, PerúBrasil-Bolivia y Andino); y en otros, en la existencia de corredores económicos ya en funcionamiento (Mercosur-Chile, de capricornio, del sur).

Por último, las áreas de influencia de los grupos de proyectos, explicitadas en la metodología del análisis de potencial de $\operatorname{IprLg}$, hacen las veces de subdivisiones territoriales operativas de los EID. Esta guía metodológica estipula que para la delimitación física de las áreas de influencia se debe dar previa cuenta de la contribución de la misma a la integración económica o de la generación de servicios logísticos de valor agregado (p. 7). Luego, se recomienda abordar la definición del área de influencia desde el enfoque clásico de las redes o sistemas de ciudades, lo cual permite identificar polos productivos y de consumo de mayor peso funcional, a partir de los que se va tejiendo una red de flujos de carga y de personas que configuran un espacio territorial interrelacionado; el conjunto que resulta de estas interacciones es el área de influencia (p. 10). Con posterioridad, siguiendo la misma guía, el planificador debe realizar un análisis del tejido productivo y sus cadenas

20 Para una lectura crítica del uso de la metáfora de las barreras (normativas y físicas) por parte del IIRSA, recomendamos Zibechi (2006). 
integrantes, con base en información secundaria recabada y entrevistas realizadas a las cámaras de productores y a fuentes del sector público (p. 11). Con esta información se examina (fase 1.3) el cúmulo de flujos sistémicos (no ocasionales) de bienes intermedios y bienes de capital que circulan entre dos países, en el sentido de exportaciones (y sus destinos) e importaciones (y sus orígenes) propias del área de influencia del Grupo de Proyectos (GP) previamente limitada. Esta lectura no incluye la totalidad de los flujos sistémicos, sino que se debe trabajar con productos cuyos flujos tienen mayor importancia en términos de valor (p. 12).

Si el enfoque liberalizador tuvo por finalidad la eliminación de todas las barreras a la circulación del capital, la arquitectura territorial propuesta por IIRSA a partir de los principios del regionalismo abierto cepalino propone una relectura del espacio donde se subdivide la integralidad transnacional latinoamericana en tres espacialidades de articulación económica: región, subregiones y microcircuitos bi- o trinacionales. 


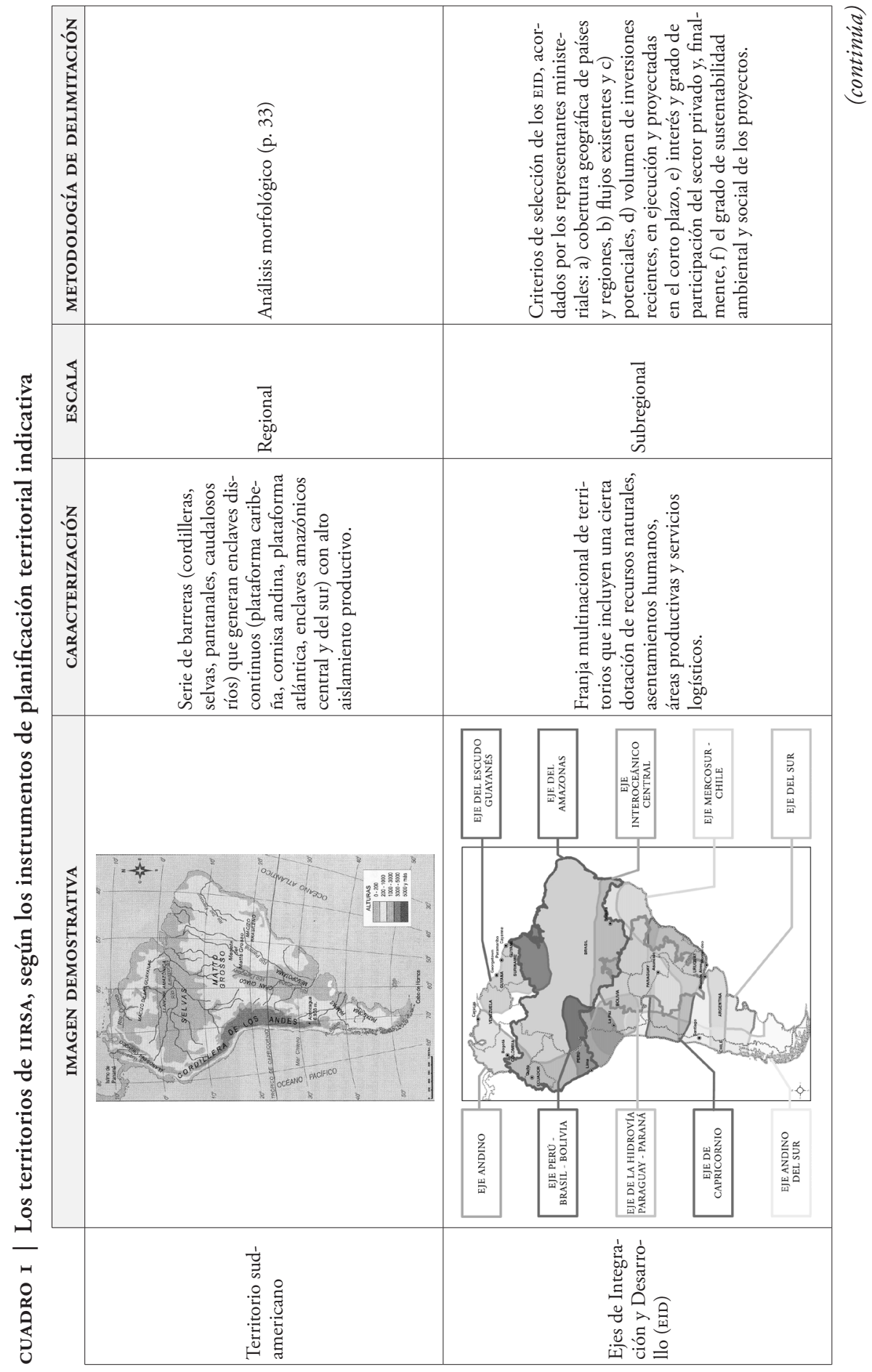




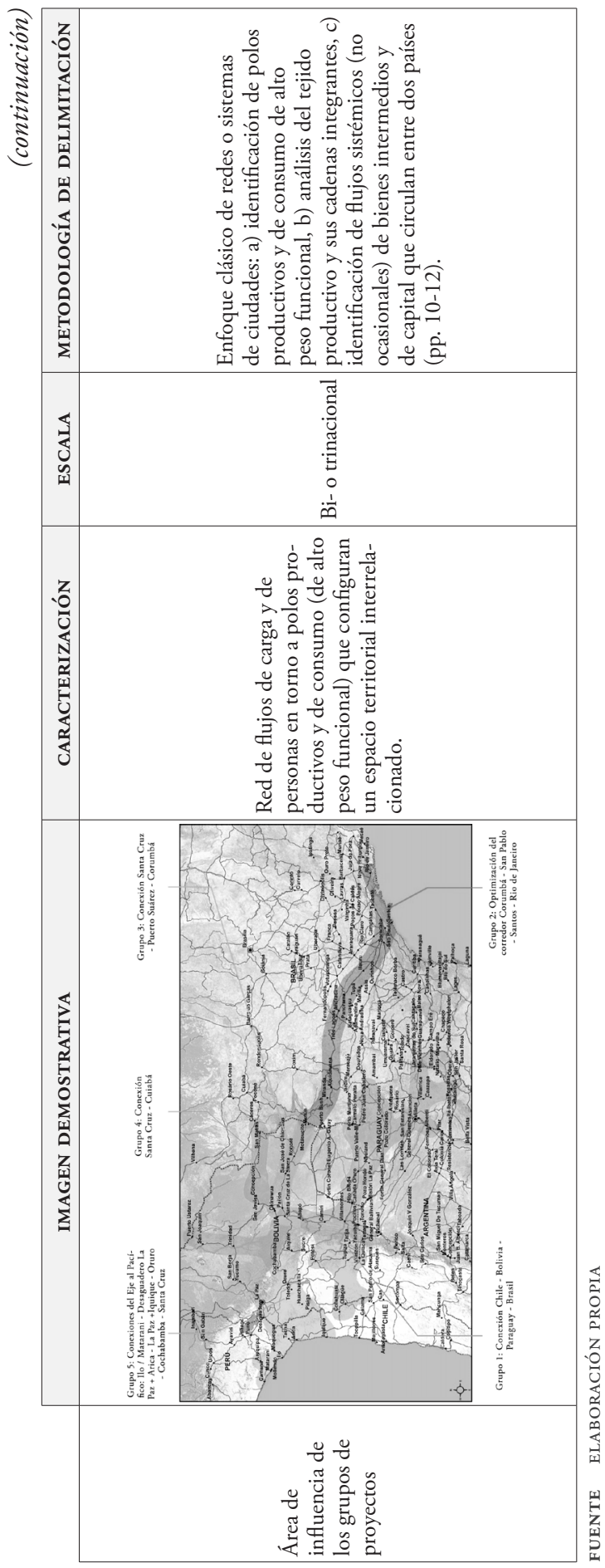




\section{Consideraciones finales y líneas de debate}

Para Zevaco y Kretchmer (2011), el objetivo principal de IIRSA es la producción de un espacio específico, por medio de la reestructuración de las relaciones socialesespaciales y su legitimación mediante un mito de desarrollo. Las autoras dirán que la territorialidad de IIRSA define a los territorios "en base a criterios netamente económicos (materia prima, actividades productivas) y lo(s) reduce a un instrumento de planificación estratégica para realizar intervenciones sobre espacios y poblaciones" (p. 179). La finalidad última, dirán Rodríguez y Alvarenque (2006), es una integración espacial "orientada a la profundización de los procesos económicos de extracción acelerada de los recursos naturales de Suramérica” (p. 22).

Se puede afirmar que desde la perspectiva de IIRSA, la maximización en el provecho de los mercados globales requiere de la aplicación de intervenciones que fortalezcan e incentiven los flujos productivos y extractivos actualmente existentes, y que su modalidad particular de intervención es desde las obras en infraestructura de conectividad. La producción del espacio, en este sentido, es interpretada como un segundo proceso de valorización del capital. Y esta segunda valorización hacia la que se hallan enfocados los objetivos de IIRSA, es también la misma que pone en primacía a la tecnocracia de planificadores regionales.

De la exposición de los distintos territorios y subterritorios de IIRSA, se destacan tres características distintivas que hacen a una definición de la territorialidad propia de IIRSA:

1. El territorio IIRSA pondera la racionalidad instrumental económica por sobre cualquier otra perspectiva de desarrollo. Nunca se hace mención a factores de orden religioso, cultural, político y/o de interés colectivo en ninguna de las tres territorialidades descritas. La consideración de patrones de sustentabilidad social o ambiental, por medio de la EASE, no se constituye en territorialidad en el esquema IIRSA debido a que (a) se realiza sobre los proyectos mismos, lo cual implica una instancia de planificación regional posterior; y (b) no ha sido utilizada en forma sistemática hasta el momento.

2. El territorio IIRSA se basa en la naturalización y generalización del actual esquema de flujos extractivos. En los tres casos se asume una naturalización de los flujos extractivos actuales (usados para la delimitación de las áreas de influencia) y potenciales (usados para la delimitación de los EID), según el actual macrocontexto de valorización financiera de las actividades extractivas en la región ${ }^{21}$.

3. El territorio IIRSA coloca al sector privado como único agente considerado y con voz. Considerado, porque sus intereses deben ser tomados en cuenta en la delimitación de los EID; y con voz, porque es -junto con el sector público- uno de los actores que la guía metodológica de análisis IprLg propone consultar para definir las áreas de influencia de los grupos de proyectos.

21 Con este argumento se deduce que la estabilidad de largo plazo de los eID depende casi íntegramente del sostenimiento del actual ritmo de producción y extracción, algo poco probable a mediano plazo debido a la degradación del suelo, al limitado stock de minerales y petróleo y a lo inestable que se ha mostrado el mercado financiero internacional en los últimos tres años. 
Aparece en todo ello una marcada dicotomía entre un discurso legitimante respecto de la planificación descentralizada y fundada en objetivos políticos de desarrollo regional y nacional, y las metodologías e instrumentos de actuación fuertemente anclados en los modelos tecnocráticos. La conceptualización de territorio de la que se nutren los planificadores regionales de IIRSA toma como base conceptual la Nueva Geografía Económica (NGE), donde priman los rendimientos decrecientes del espacio, al tiempo que discursivamente se explicita la adhesión a las nociones de planificación por consenso.

Se propone conceptualizar a los planificadores regionales de IIRSA como detentores de poder tecnocrático ${ }^{22}$, por dos razones: a) porque su funcionalidad global representa una continuidad diferencial del rol asumido por la tecnocracia de economistas neoliberales, y b) porque la práctica de efectivización de su rol implica necesariamente la reproducción de un saber técnico (know how) especializado, generalizable, explícito y sistematizado.

La tecnocracia de planificadores regionales de IIRSA se funda en las ideas del regionalismo abierto cepalino, se encuentra principalmente integrada por técnicos miembros de la сСт y algunos consultores privados, y ha asumido el rol de articuladora entre estos objetivos estratégicos de los Estados y su aplicabilidad. Para estas finalidades, los planificadores despliegan todo un abanico de actividades, las cuales abarcan desde la diagramación de los proyectos y su articulación en torno a los principios amplios del regionalismo abierto y las legislaciones nacionales, hasta el proceso de marginación y eliminación de racionalidades, estrategias e identidades diferenciales presentes en los territorios de intervención. Se propone diferenciar estas actividades, por un lado, en la construcción de territorialidad (como las prácticas de definición, caracterización y delimitación de los territorios) y, por otro, en la construcción de consenso (como las prácticas de selección y retención diferencial de discursos, identidades y prácticas). En ambos casos se percibe una fuerte concentración del poder de toma de decisión en la tecnocracia de los planificadores regionales, permeables y susceptibles a los intereses e influencias de los sectores económicos imperantes, siendo tales procesos intermediados por los Estados nacionales.

Los planificadores regionales de IIRSA han venido llevado a cabo prácticas de selección, retención y reproducción de discursividades y racionalidades particulares (Jessop, 2007) en sus actividades de planificación, procurando apoderarse de la idea de un supuesto consenso por medio de sus herramientas científico-técnicas (PTI), y haciendo uso de las mismas como dispositivos de despolitización y naturalización de los imperativos económicos (Ciuffolini \& De la Vega, 2011).

Esta concentración de poder decisorio en los planificadores regionales de IIRSA genera un complejo problema de legitimidad política en los Estados nacionales, resultando en un vacío democrático en la medida en que decisiones políticas de gran envergadura son transferidas a instancias técnicas supranacionales, sin los correspondientes mecanismos efectivos de control y participación civil nacional.

22 Videla, Gasparotto y Nardi (2010) asocian al вм у al BID con la metáfora de arquitectos de las geografías sociopolíticas requeridas por los imperativos del capital, debido a que en estas instituciones se da la combinación de los atributos de actores constructores y poseedores de saberes técnicos (p. 4). 
Los planos y proyectos carecen no solo de participación política efectiva en su elaboración, sino también de oportunidades de discusiones públicas en los diferentes países. Se observa una reproducción de un padrón muy común a la burocracia latinoamericana, en que esta asume el papel de mediadora de conflictos sociales, pero con accountability extremamente limitada (Nef, 2010, p. 520).

Se hace posible proponer la hipótesis de que la tendencia burocrática al aislamiento, que de un lado favoreció la emergencia de una dimensión latinoamericana del desarrollo como alternativa al enfoque liberalizador y desregulador de los años ochenta, ha conllevado, de otro, un distanciamiento de los planificadores respecto de las realidades y disputas sociales existentes. Esta distancia ha subrayado la influencia de los sectores económicos dominantes (representados por los consultores privados encargados de la elaboración de las visiones de negocios y los $\operatorname{IprLg}$ ) sobre estas realidades conflictivas. La resultante es una concepción de desarrollo que refuerza a los sectores extractivo-exportadores en detrimento de concepciones alternativas, aislando y buscando eliminar a amplios sectores sociales en resistencia.

\section{Documentos oficiales IIRSA revisados}

Análisis del Potencial de Integración Productiva y Desarrollo de Servicios Logísticos de Valor Agregado de Proyectos IIRSA (IprLg). Guía metodológica revisada. M. Barceló Koser y R. Barcia Fonseca, preparado para el Instituto para la Integración de América Latina y el Caribe (BID/INTAL) en el marco de la Iniciativa IIRSA (Buenos Aires, julio de 2009). Disponible en http://www10.iadb.org/intal/intalcdi/PE/2010/04630.pdf

Análisis del potencial IprLg, aplicación al grupo 3 de proyectos del EID de Capricornio. Resumen ejecutivo (Asunción, Paranaguá, julio 2009). Disponible en http://www10.iadb.org/ intal/intalcdi/PE/2010/04633.pdf

"Comunicado de Brasilia”. Reunión de Presidentes de América del Sur. Brasilia, 1 de septiembre de 2000. Disponible en http://www.iirsa.org/admin_iirsa_web/Uploads/Documents/ comunicado_brasilia_esp.pdf

IIRSA 10 AÑOS DESPUÉS: Sus logros y desafíos. Buenos Aires: BID/INTAL, 2011. Disponible en http:// www.iirsa.org/Document/Detail?Id=3356

Metodología de Evaluación Ambiental y Social con Enfoque Estratégico (EASE). Caracas: Dirección de Medio Ambiente/Corporación Andina de Fomento (DMA/CAF), julio 2009. Disponible en http://www.iirsa.org/admin_iirsa_web/Uploads/Documents/ ease_metodologia_iirsa.pdf

"Plan de Acción para la Integración de la Infraestructura Regional de América del Sur". Reunión de Ministros de Trasporte, Telecomunicaciones y Energía de América del Sur (Montevideo, 4 y 5 de diciembre de 2000). Disponible en http://www.iirsa.org/Page/ Detail?menuItemId $=53$

Visión de Negocios del Eje de Capricornio. Argentina, Bolivia, Brasil, Chiley Paraguay. Acutalización 2007. Buenos Aires, diciembre de 2007. Disponible en http://www.iirsa.org/admin iirsa_web/Uploads/Documents/cad_vn_2007_competo.pdf 


\section{Referencias bibliográfícas}

Abrucio, F.L., Pedroti, P. \& Pó, V.M. (2010). A formação da burocracia brasileira: a trajetória e o significado das reformas administrativas. En M.R. Loureiro, F.L. Abrucio \& R. S. Pacheco (Eds.), Burocracia e politica no Brasil: desafios para a ordem democrática no século XXI (pp. 27-71). Río de Janeiro: FGV Editora.

Benko, G. (2002). Economia, espaço e globalizaçäo na aurora do século XXI. San Pablo: HUCITEC. Brenner, N. (2003). 'Glocalization' as a state spatial strategy: urban entrepreneurialism and the new politics of uneven development in Western Europe. En J. Peck \& H. Yeung (Eds.), Remaking the Global Economy: Economic-Geographical Perspectives (pp. 197216). Londres: Sage. doi: http://dx.doi.org/10.4135/9781446216767.n12

Ciuffolini, M.A. \& De la Vega, C. (2011). Conflictos ambientales en América Latina: un desafío teórico y práctico [En línea]. Documento presentado en XXVIII Congresso Internacional da Alas, 6 a 11 de setembro de 2011, ufpe, Recife-PE. Disponible en http://goo.gl/feiKJ

Cocco, G., Monié, F. \& Silva, G. (2000). Brasil em Ação. Sistema de transportes e inserciōn competitiva de Brasil en el proceso de globalización. Río de Janeiro: Ediciones do LAB TEC.

De Mattos, C.A. (2004). De la planificación a la governance: implicancias para la gestión territorial y urbana. Revista Paranaense de Desenvolvimento, (107), 9-23. En http:// www.ipardes.pr.gov.br/ojs/index.php/revistaparanaense/article/view/99

Frey, K. (2008). Development, good governance and local democracy. Brazilian Political Science Review, 2(2), 39-73. Disponible en http://socialsciences.scielo.org/scielo. php?pid=S1981-38212008000100007\&script=sci_arttext

Gudynas, E. (2005). El 'regionalismo abierto' de la Cepal: insuficiente y confuso [En línea]. Silver City, NM: International Relations Center. Disponible en http://www. integracionsur.com/americalatina/GudynasRegionalismoAbiertoIrc.pdf

Gudynas, E. (2009). Diez tesis urgentes sobre el nuevo extractivismo. Contextos y demandas bajo el progresismo sudamericano actual. En AaVv, Extractivismo, politica y sociedad. (pp. 187-225). Quito: Centro Andino de Acción Popular (CAAP) y Centro Latino Americano de Ecología Social (CLAES).

Fujita, M. \& Krugman, P. (2004). The new economic geography: Past, present and the future. Papers in regional Science, 83(1), 139-164. doi: 10.1007/s10110-003-0180-0

Harvey, D. (2005). El "nuevo" imperialismo: acumulación por desposesión. En L. Panitch \& C. Layes (Eds.), Socialist Register 2004 El nuevo desafío imperial (pp. 99-129). Buenos Aires: Consejo Latinoamericano de Ciencias Sociales (CLACso). Disponible en http:// biblioteca.clacso.edu.ar/gsdl/collect/clacso/index/assoc/D8555.dir/harvey.pdf

Jessop, B. (2007). Análisis semiótico crítico y economía político-cultural. En B. Jessop, Capitalismo(s): Discurso y materialidad en las formaciones sociales capitalistas contemporáneas (pp. 77-107). Córdoba: Ediciones de la Universidad Católica de Córdoba.

Jessop, B. (2008). The development of the strategic-relational approach. En B. Jessop, State Power: A strategic-relational approach (pp. 21-53). Cambridge: Polity. 
Kuwahara, N., Oliveira Gomes, H. \& Santos, M.P. (2008). Análise e proposição de modelo de apoio ao planejamento de investimentos em infra-estrutura de transportes no Brasil. Manaos: Companhia Brasileira de Trens Urbanos (свтU).

Mancano Fernández, B. (2008). Sobre a tipologia de territórios [En línea]. Disponible en http:// acciontierra.org/IMG/pdf/BERNARDO_TIPOLOGIA_DE_TERRITORIOS.pdf

Nef, J. (2010). Administração pública e reforma do setor público na América Latina. En M.R. Laureiro, F.L. Abrucio \& R.S. Pacheco (Eds.), Burocracia e política no Brasil: desafios para a ordem democrática no século XXI (pp513-535). Río de Janeiro: Fundaçáo Getulio Vargas.

Rodríguez, M. \& Alvarenque, I. (2006). Las espacialidades abiertas de América Latina. Otro análisis crítico al ordenamiento territorial de la iniciativa IIRSA. Tesina de la carrera de posgrado Especialización en Educación en Ambiente para el Desarrollo Sustentable, Escuela Pedagógica y Sindical "Marina Vilte" de la Confederación de Trabajadores de la Educación de la República Argentina (CTERA) y Universidad Nacional de Comahue, Neuquén, Argentina.

Silva, P. (1997). Ascenso tecnocrático y democracia en América Latina. Revista Nueva Sociedad, (152), 68-77. Disponible en http://nuso.org/upload/articulos/2640_1.pdf

Silva, P. (2010). Tecnocracia y gobernabilidad democrática en América Latina [En línea]. San José, Costa Rica: Facultad Latinoamericana de Ciencias Sociales (fLACso). Disponible en http://www.plataformademocratica.org/Publicacoes/19466.pdf

Svampa, M. (2008). La disputa por el desarrollo: territorio, movimientos de carácter socioambiental y discursos dominantes [En línea]. Disponible en http://www.extractivismo. com/documentos/SvampaSobreDesarrollo.pdf

Videla, G., Gasparotto, M. \& Nardi, M.A. (2010). ¿Arquitectos del mundo? Acerca de la centralidad del Banco Mundial y el Banco Interamericano de Desarrollo en la planificación territorial desde 1990 [En línea]. Scripta Nova. Revista Electrónica de Geografia y Ciencias Sociales, 14(331) (15). Disponible en http://www.ub.es/geocrit/ sn/sn-331/sn-331-15.htm

Zevaco, S. \& Kretchmer, R. (2011). Neoliberalismo y políticas de infraestructura. En L. Rojas Villagra (Comp.), La economía paraguaya bajo el orden neoliberal (pp. 141-193). Asunción: Base Investigaciones Sociales (Base-Is).

Zibechi, R. (2006, 13 de junio). IIRSA: la integración a la medida de los mercados. Programa de las Américas, Informe Especial. Silver City, NM: International Relations Center. Disponible en http://www.alternative-regionalisms.org/wp-content/ uploads/2009/07/zibechi-iirsa.pdf

Zibechi, R. (2012). Brasil potencia. Entre la integración regional y un nuevo imperialismo. Bogotá: Ediciones desde abajo. Disponible en http://goo.gl/YdNgPF 
TRIBUNA 